\title{
Estimation in situ chez le ruminant de la valeur azotée du lupin en fonction de la qualité du broyage et de la taille des particules
}

\author{
AR Kibelolaud, M Vernay *, C Bayourthe, R Moncoulon, P Cros
}

\author{
ENSA, laboratoire d'ingenierie agronomique, \\ 145 avenue de Muret, 31076 Toulouse Cedex
}

(Reçu le 12 mars 1991; accepté le 7 août 1991)

\begin{abstract}
Résumé - Pour estimer la valeur azotée (PDI) de la graine de lupin en fonction de la qualité du broyage (grossier ou fin) et de la taille des particules, 4 vaches taries, canulées ont été utilisées. La méthode des sachets de nyion a été retenue afin d'évaluer les disparitions ruminale et intestinale de l'azote (N). La solubilité de l'azote in vitro a été mesurée. Une bonne corrélation est observée entre cette solubilité et la dégradabilité ruminale de l'azote $(2 \mathrm{~h})$ d'une part et entre la solubilité et le coefficient a des cinétiques de dégradation de l'azote d'autre part. Un broyage grossier (grille $5 \mathrm{~mm}$ ) réduit la solubilité de l'azote en salive artificielle : 35,6 vs 75,5 et $78,8 \%$ (grilles 1,5 et $1 \mathrm{~mm}$ ) et la dégradabilité de l'azote ( $D g N$ ) dans la panse; les valeurs de $D g N$ obtenues sont : 62,5 vs 93,0 et $94,9 \%$ respectivement (taux de passage théorique des particules de $0,06 / \mathrm{h}$ ). La disparition totale de l'azote alimentaire dans le tractus digestif étant toujours élevée : $99,8 \%$, la fraction qui disparaît lors du transit intestinal augmente avec la grille de broyage. Les valeurs estimées de PDI ( $g / \mathrm{kg} \mathrm{MS}$ ) pour un broyage fin à la grille de $1 \mathrm{~mm}$ sont : 232 (PDIN) et 91 (PDIE); un broyage aux grilles de 1,5 et 5 $\mathrm{mm}$ élève ces teneurs de 1,7 et $25,4 \%$ (PDIN) et de 7,8 et $140,6 \%$ (PDIE) respectivement. Lorsque la taille des particules est prise en considération, les résultats obtenus confirment les précédents.
\end{abstract}

lupin / broyage / vache / taille particule / sachet nylon / valeur PDI

Summary - Estimation of the protein value of finely or coarsely milled lupin seeds. Samples of Lupinus albus cv Lublanc were ground to pass either through a 1.0, a 1.5- $\mathrm{mm}$ (fine) or a 5-mm (coarse) screen and particle sizes were measured. The effect of milling the seeds and of particle size on in situ nitrogen $(N)$ degradability $(\mathrm{Dg})$ was measured by the nylon bag technique using fistulated non-lactating Holstein cows. Animals were fed a ration of $30 \%$ soybean meal and Italian ryegrass hay. To estimate rumen degradation of $N$, nylon bags were placed in the rumen for $2,4,8,16$, 24 and $48 \mathrm{~h}$. The effective DgN was calculated assuming a rumen outflow rate of $0.06 / \mathrm{h}$. To evaluate feed-indigestible $N$, bags were left in the rumen for $16 h$, placed in a pepsin bath for $2 h$ and then introduced into the duodenum for subsequently recovery in the feces. The PDI contents of the samples tested were calculated using DgN and fermentable organic matter (French PDI system). The in vitro $N$ solubility of the same samples was also measured. Significant correlations were found between $N$ solubility and the in situ rumen degradability of $N(2 h)$, and between $N$ solubility and the coefficient a of degradability kinetics in situ. The effective $\mathrm{DgN}$ values for coarse and fine samples of lupin seeds were: 62.5, 93.0 and 94.9\% respectively. The mean value for whole tract digestion of $N$ was always high $(99.8 \%)$. Grinding the lupin coarsely decreased ruminal $D g N$, with a corresponding

\footnotetext{
* Correspondance et tirés à part
} 
increase in the amounts digested in the intestine; intestinal disappearance of $N$ for coarse and fine samples of lupin was 37.3, 6.8 and $4.9 \%$. The PDIN and PDIE contents $(\mathrm{g} / \mathrm{kg} \mathrm{DM}$ ) for lupin ground finely enough to pass through a $1-\mathrm{mm}$ screen were 232 and 91 respectively; milling the lupin to pass through 1.5 or $5.0-\mathrm{mm}$ screen increased these values by 1.7 and $25.4 \%$ for PDIN and by 7.8 and $140.6 \%$ for PDIE. The differences in values obtained from coarse and fine lupin samples were also confirmed when the effects of substrate particle size were tested.

lupin / grinding / nylon bag / protein value / particle size / cow

\section{INTRODUCTION}

Les vaches à forte production laitière ont des besoins élevés en acides aminés. Or, dans le rumen, les enzymes bactériennes dégradent une partie des protéines alimen-taires, ce qui en réduit l'efficacité lorsqu'il s'agit d'un supplément azoté $(\mathrm{N})$ de bonne qualité. L'importance de cette digestion est fonction de la dégradabilité des matières azotées (MA) de l'aliment, de l'intensité de l'activité des microorganismes et du temps de séjour dans la panse. Par ailleurs, de nombreux travaux ont mis l'accent sur l'importance de la taille des particules lors de la dégradation des protéines alimentaires dans le rumen. Les effets d'une réduction de la taille des particules sont de deux ordres :

- une augmentation du rapport (surface d'attaque / masse), ce qui favoriserait les actions bactériennes :

- une réduction du temps de séjour dans le rumen ce qui pourrait occasionner une diminution de la dégradation des MA (Nordin et Campling, 1976; Galyean et al, 1979, 1981; Thomson et Beever, 1980; Eliman et Ørskov, 1984; 1985; Thomas et al, 1988; Belyea et al, 1989). Si de nombreuses expérimentations ont été consacrées à l'étude de la dégradabilité ruminale et de la digestibilité intestinale des MA des tourteaux, des céréales (avoine, blé, maïs) et des graines entières oléoprotéagineuses (colza, soja), il n'en a pas été de même en ce qui concerne la graine de lupin. Or cette légumineuse est particulièrement riche en MA : $32-36 \%$ de la matière sèche (MS) et en lipides : 7-12\% MS (Hove et King, 1978; Guillaume et al, 1987; Valentine et Bartsch, 1988), aussi pourrait-elle être substituée aux tourteaux et graines de soja dans l'alimentation des vaches laitières si ses protéines constitutives étaient moins rapidement dégradées lors de leur séjour dans les réservoirs de fermentation. En effet, pour la graine du lupin broyée à la grille de $0,8 \mathrm{~mm}$, des valeurs élevées de dégradabilité théorique de l'azote $(93,4-95,0 \%)$ sont mentionnées par différents auteurs (Émile et al, 1988; Andrieu et al, 1988).

Le but de ce travail a été d'étudier l'influence du broyage (grossier et fin) et de la taille des particules sur la digestion des MA du lupin, in sacco dans le rumen et dans les régions postérieures du tube digestif.

\section{MATÉRIEL ET MÉTHODES}

\section{Animaux}

Quatre vaches laitières de race Holstein, taries, pesant environ $600 \mathrm{~kg}$ et munies de canules (ruminale, duodénale) ont été utilisées. Les animaux ont reçu journalièrement, en 2 repas égaux ( 8 et $16 \mathrm{~h}$ ), $10 \mathrm{~kg}$ de MS d'une ration constituée de $70 \%$ de foin de rye-grass et de $30 \%$ de tourteau de soja. 


\begin{abstract}
Aliments
Des graines de lupin (Lupinus albus cv Lublanc) broyées à la grille de 5,1 et $1,5 \mathrm{~mm}$ ont été tamisées par voie humide selon la technique de Grenet (1970). Les tamis utilisés avaient une ouverture de maille comprise entre 1 et $0,10 \mathrm{~mm}$; le poids des particules recueillies sur les différents tamis a été exprimé en pourcentage du poids total.
\end{abstract}

\section{Dégradation ruminale de l'azote in sacco}

Des sachets de nylon* soudés $(11 \times 6 \mathrm{~cm}$; de taille de pore $46 \mu \mathrm{m} ; 3 \mathrm{~g}$ d'échantillon) ont séjourné dans le rumen pendant $2,4,8,16,24$ et $48 \mathrm{~h}$. Après incubation, ils ont été lavés et séchés (Michalet-Doreau et al, 1987). Pour chaque échantillon, 72 sachets ont été préparés (3 sachets par temps de séjour et par vache) et répartis entre les 4 vaches.

\section{Digestion intestinale de l'azote en sachets mobiles}

Les sachets de nylon * $(6 \times 6 \mathrm{~cm}$; taille de pore $46 \mu \mathrm{m} ; 1,5 \mathrm{~g}$ d'échantillon) incubés dans le rumen durant $16 \mathrm{~h}$, ont été ensuite immergés pendant $2 \mathrm{~h}$ dans une solution de pepsine- $\mathrm{HCl}$ (pH 2) maintenue à $38^{\circ} \mathrm{C}\left(3 \mathrm{~g}\right.$ de pepsine ${ }^{* *} / 1$; 30 sachets $/ \mathrm{l})$, puis introduits $1 \mathrm{~h}$ après le repas du soir dans lintestin, via la canule duodénale (15 sachets $j^{-1}$ par vache). Les sachets ont été recueillis dans les fèces dès le lendemain matin; ceux ayant transité plus de $24 \mathrm{~h}$ ont été éliminés. Dès leur récupération, les sachets ont été lavés et séchés (Peyraud et al, 1988). Seize sachets ont été préparés par échantillon et répartis entre les 4 vaches.

\section{Analyses}

Pour les échantillons de lupin, les teneurs en MS, matière organique (MO), MAT, matières

\footnotetext{
${ }^{*}$ Blutex $\mathrm{T}_{50}$ (Tripette et Renaud; Paris)

** Pepsine porcine A (600 à 1000 Ul/mg); Sigma
}

grasses (MG) et cellulose brute (CB) ont été déterminées selon les méthodes décrites dans Anonyme (1971). Par ailleurs nous avons évalué la solubilité de l'azote en salive artificielie d'après la technique préconisée par Vérité et Demarquilly (1978).

\section{Calculs}

Afin de calculer la dégradabilité de l'azote alimentaire dans le rumen, les résultats de dégradation par aliment ont été ajustés au modèle exponentiel décrit par Ørskov et McDonald (1979), à savoir :

$D(t)=a+b\left(1-e^{-c t}\right)$ qui suppose 3 fractions azotées : une fraction immédiatement dégradable (a); une autre dont la dégradation se fait plus lentement (b) avec une vitesse (c) qui décroît exponentiellement et une fraction non dégradable $(100-a-b)$. Dans le but de comparer la dégradabilité de l'azote des échantillons de lupin dans la panse, un taux de sortie des particules alimentaires de $0,06 / \mathrm{h}$ a été retenu (Vérité et al, 1987) et la dégradabilité de l'azote (DgN) a été obtenue à l'aide de la formule suivante : $D g N=a+(b c) /(c+0,06)$.

Chez le ruminant, l'estimation de la valeur azotée réelle des aliments est complexe car, au cours de sa digestion, l'animal tire profit des formes azotées simples transformées en acides aminés par les microorganismes de la panse. Cette valeur azotée peut cependant être évaluée à partir du système $P D I$ (protéines réellement digestibles dans l'intestin). Les valeurs $P D I$ ont été estimées d'après les équations de base proposées par Vérité et al (1987) en utilisant : la teneur en MA, la $D g N$, la digestibilité apparente dans l'intestin des MA non dégradées dans le rumen et la teneur en MO fermentescible (MOF); elle même dérivée de la teneur en MO digestible et de la composition chimique. La valeur de la digestibilité des MO du lupin donnée dans les tables de l'INRA (Andrieu et al, 1988) a été utilisée pour la détermination des quantités de MO digestibles.

Chaque donnée analytique représente la moyenne arithmétique des échantillons affectée de l'écart type. Les effets du broyage à différentes grilles, d'une part, et de la taille des parti- 
cules, d'autre part, ont été soumis à une analyse de variance et les moyennes comparées à l'aide du test de Newman-Keuls au seuil de 5\% (Dagnélie, 1975). Les coefficients de régression (r) ou de détermination $\left(r^{2}\right)$ ont été mis en évidence pour chaque mode de broyage et pour chaque taille de particules afin de déterminer :

- les niveaux de signification du modéle d'équation (linéaire) entre la solubilité de l'azote et la dégradation ruminale in sacco $(2 \mathrm{~h})$. La solubilité de l'azote et le coefficient a des cinétiques de dégradation de l'azote,

- les niveaux de signification du modèle d'équation (exponentiel) entre la disparition in sacco de l'azote dans le rumen et le temps d'incubation.

\section{RÉSULTATS}

\section{Granulométrie - Composition chimique}

De l'analyse du tableau I, il ressort que la taille moyenne des particules du lupin diminue significativement avec le diamètre de la grille de broyage. C'est ainsi que $87 \%$ des particules ont une taille supérieure à $1 \mathrm{~mm}$ après un broyage grossier, alors que 76,4 et $90 \%$ des particules ont une taille comprise entre 0,75 et $0,10 \mathrm{~mm}$ si les grilles utilisées sont de 1,5 et $1 \mathrm{~mm}$ respectivement. La nature du broyage ne modifie pas la composition chimique de la MS (tableau II).

\section{Solubilisation de l'azote in vitro}

Si la concentration en $\mathrm{N}$ n'est pas significativement influencée par le mode de broyage du lupin, il n'en est pas de même pour sa solubilité. Cette dernière est particulièrement élevée lorsque le lupin est broyé aux grilles de 1 ou $1,5 \mathrm{~mm}$ soit : 78,8 et $75,5 \%$ respectivement; la valeur obtenue après un broyage grossier étant de $35,6 \%$ seulement. La solubilité de l'azote varie inversement à la taille (Tmm) des particules, eg, 88,6\% $(0,10<T<$ $0,25), 76,7 \%(0,25<T<0,75), 65,9 \%$ $(0,75<T<1)$ et $40,8 \%(T>1)$.

\section{Digestion ruminale de.l'azote in sacco}

La digestion des MA du lupin par les microorganismes ruminaux est influencée par la nature du broyage et la taille des particules (fig 1). Au cours du séjour dans les réservoirs de fermentation, les MA du lupin broyé à la grille de $5 \mathrm{~mm}$ sont lente-

Tableau I. Granulométrie (\%) du lupin.

\begin{tabular}{|c|c|c|c|}
\hline \multirow{2}{*}{$\begin{array}{l}\text { Tailles des particules } \\
\text { (Tmm) }\end{array}$} & \multicolumn{3}{|c|}{ Grilles de broyage $(\mathrm{mm})$} \\
\hline & 5,0 & 1,5 & 1,0 \\
\hline $\begin{aligned} 1,00 & <T \\
0,75 & <T<1,00 \\
0,25 & <T<0,75 \\
0,10 & <T<0,25 \\
& T<0,10\end{aligned}$ & $\begin{array}{r}87,4^{a} \pm 1,2 \\
3,2^{b} \pm 0,2 \\
5,8^{c} \pm 0,3 \\
2,2^{c} \pm 0,1 \\
0,9^{b} \pm 0,1\end{array}$ & $\begin{array}{r}2,1^{b} \pm 0,1 \\
10,2^{a} \pm 0,7 \\
43,7^{a} \pm 1,0 \\
32,7^{c} \pm 1,7 \\
8,8^{a} \pm 2,8\end{array}$ & $\begin{array}{r}- \\
1,5^{\mathrm{c}} \pm 1,2 \\
13,1^{\mathrm{b}} \pm 2,5 \\
76,8^{\mathrm{a}} \pm 1,8 \\
8,6^{\mathrm{a}} \pm 1,7\end{array}$ \\
\hline
\end{tabular}

Moyennes et écart type; les valeurs moyennes sur une même ligne affectées d'un même exposant ne sont pas significativement différentes. 
Tableau II. Composition chimique du lupin (\% MS).

\begin{tabular}{|c|c|c|c|}
\hline \multirow{2}{*}{ Composant * } & \multicolumn{3}{|c|}{ Grilles de broyage $(\mathrm{mm})$} \\
\hline & 5,0 & 1,5 & 1,0 \\
\hline MO & 95,0 & 95,6 & 95,5 \\
\hline $\operatorname{MAT}(6,25)$ & 38,8 & 38,9 & 38,8 \\
\hline MG & 9,1 & 8,7 & 8,7 \\
\hline $\mathrm{CB}$ & 10,7 & 10,8 & 10,7 \\
\hline
\end{tabular}

- MO : matière organique; MAT : matière azotée totale; MG : matiere grasse; $\mathrm{CB}$ : cellulose brute.

ment dégradées, ce qui n'est plus le cas après un broyage fin (fig 1a). $\AA 2 \mathrm{~h}$, puis $24 \mathrm{~h}$ d'incubation la disparition de l'azote des sachets de nylon est respectivement de 45,0 et $92,2 \%$ pour le broyage grossier; les valeurs moyennes correspondantes pour un broyage fin étant de 89,4 et $98,0 \%$. De même, une réduction de la taille des particules accroît la dégradabilité des MA (fig 1b). C'est ainsi qu'après $2 \mathrm{~h}$ d'incubation dans la panse, $54,6 \%$ de l'azote contenu initialement dans les particules de tailles supérieures à $1 \mathrm{~mm}$ a disparu; cette valeur est augmentée de $47,6 \%$ environ, lorsque les tailles s'échelonnent de 1 à $0,25 \mathrm{~mm}$ et de $67,0 \%$ pour les particules plus fines, c'est-à-dire comprises entre 0,25 et $0,10 \mathrm{~mm}$. Par la suite ces différences s'estompent progressivement, si bien qu'après $24 \mathrm{~h}$ de séjour dans le rumen elles ne sont plus significatives.

Les paramètres des cinétiques de dégradation de l'azote et les valeurs de dé-

(a)

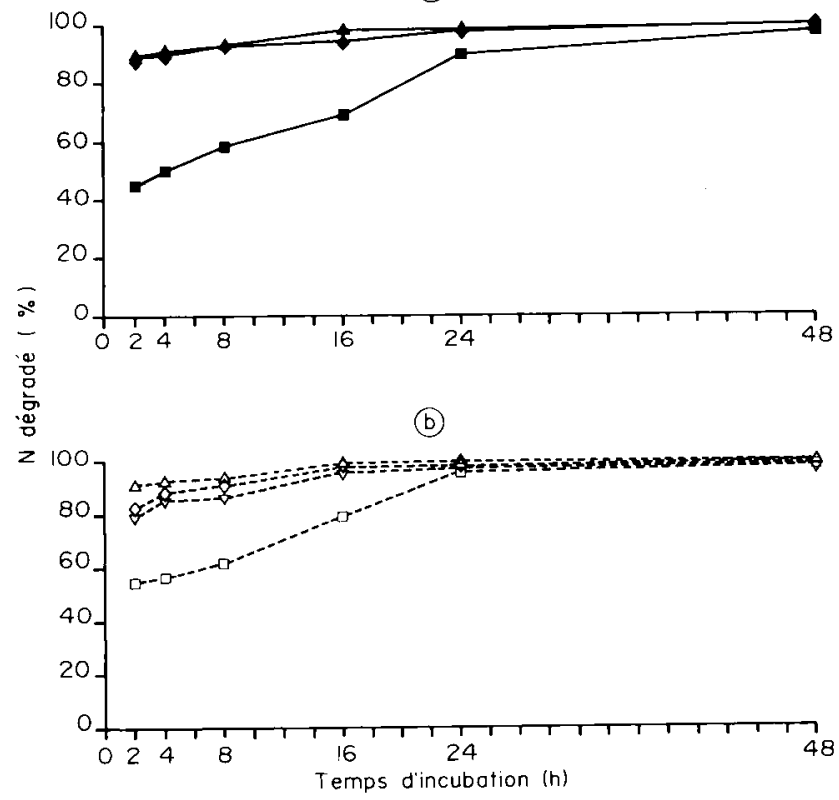

Fig 1. Dégradation de l'azote $(N)$ du lupin dans le rumen en fonction de la grille de broyage (a) : 5 $(-\square), 1,5(--)$ et $1 \mathrm{~mm}\left(-\Delta_{-}\right)$et de la taille des particules (b) : T>1,00 (- $\left.\square-\right), 0,75<T<1,00(-x-)$, $0,25<T<0,75(-0-)$, et $0,10<T<0,25 \mathrm{~mm}(-\Delta-)$. 
gradabilité ruminale $(D g N)$ sont consignés dans le tableau III. Quel que soit l'effet étudié (broyage; taille des particules), les coefficients de détermination $\left(r^{2}\right)$ sont élevés. Le lupin broyé grossièrement a une $D g N$ de $62,5 \%$ seulement; des valeurs nettement plus importantes sont obtenues avec les 2 autres grilles de broyage, soit une augmentation de 48,8 à $51,8 \%$ (grilles 1,5 et $1 \mathrm{~mm}$ ). Cette hausse est due essentiellement à l'accroissement de la fraction azotée rapidement dégradable (paramètre a) d'une part, et de la vitesse de dégradation (paramètre $c$ ) de la fraction azotée lentement dégradable d'autre part. Si la taille des particules est prise en considération, les valeurs de $D g N$ s'étendent de $75,9(T>1)$ à $96,4 \%(0,10<T<0,25)$.

Par ailleurs, il existe une relation linéaire (fig 2) significative entre la solubilité de l'azote en salive artificielle et la dégradation in sacco de l'azote (2 h) d'une part, et entre la solubilité de l'azote et le coefficient $a$ des cinétiques de dégradation de l'azote d'autre part.

\section{Digestion intestinale de l'azote en sachets mobiles}

Quel que soit l'échantillon testé, la disparition des MA dans l'ensemble du tube digestif est particulièrement élevée, soit en moyenne $99,8 \%$ (fig 3 ). En conséquence, la part d'azote d'origine alimentaire qui atteint l'intestin grêle et qui disparaît au cours du transit dans les régions postruminales est significativement plus importante lorsque le lupin est broyé grossièrement : 37,3 vs 6,8 et $4,9 \%$ (grilles 1,5 et $1 \mathrm{~mm}$ ). Les digestibilités intestinales correspondantes sont de : $99,5,97,1$ et $96,1 \%$ (valeurs utilisées pour le calcul des teneurs $P D I$ ). Lorsque la taille des particules est supérieure à $1 \mathrm{~mm}$, la disparition des MA au cours du transit intestinal est de $23,9 \%$, avec une digestibilité apparente de $99,2 \%$. Pour les particules plus fines, les valeurs correspondantes sont : 10,8 et $98,2 \%$ $(0,75<T<1), 7,6$ et $97,4 \%(0,25<T$ $0,75), 3,4$ et $94,4 \%(0,10<T<0,25)$.

Tableau III. Coefficients des cinétiques de dégradation et valeurs de dégradabilité de l'azote ( $D g N)$ en fonction de la grille de broyage et de la taille des particules de lupin.

\begin{tabular}{lccccc}
\hline Paramètres & $a$ & $b$ & $c$ & $r^{2}$ & DgN \\
\hline Grilles de broyage $(\mathrm{mm})$ & & $\%$ & & & $\%$ \\
5,0 & 28,4 & 68,6 & 5,9 & 0,88 & 62,5 \\
1,5 & 76,9 & 22,3 & 15,8 & 0,88 & 93,0 \\
1,0 & 80,5 & 18,5 & 21,2 & 0,89 & 94,9 \\
Tailles des particules T(mm) & & & & & \\
$1,00<\mathrm{T}$ & 30,0 & 67,9 & 12,6 & 0,88 & 75,9 \\
$0,75<\mathrm{T}<1,00$ & 66,2 & 32,1 & 14,8 & 0,97 & 89,0 \\
$0,25<\mathrm{T}<0,75$ & 75,1 & 23,9 & 15,1 & 0,91 & 92,2 \\
$0,10<\mathrm{T}<0,25$ & 85,7 & 13,8 & 20,7 & 0,95 & 96,4 \\
\hline
\end{tabular}

$a, b$ et $c$ : coefficients des cinétiques de dégradation (cf $p$ 249)

$r^{2}:$ coefficient de détermination. 

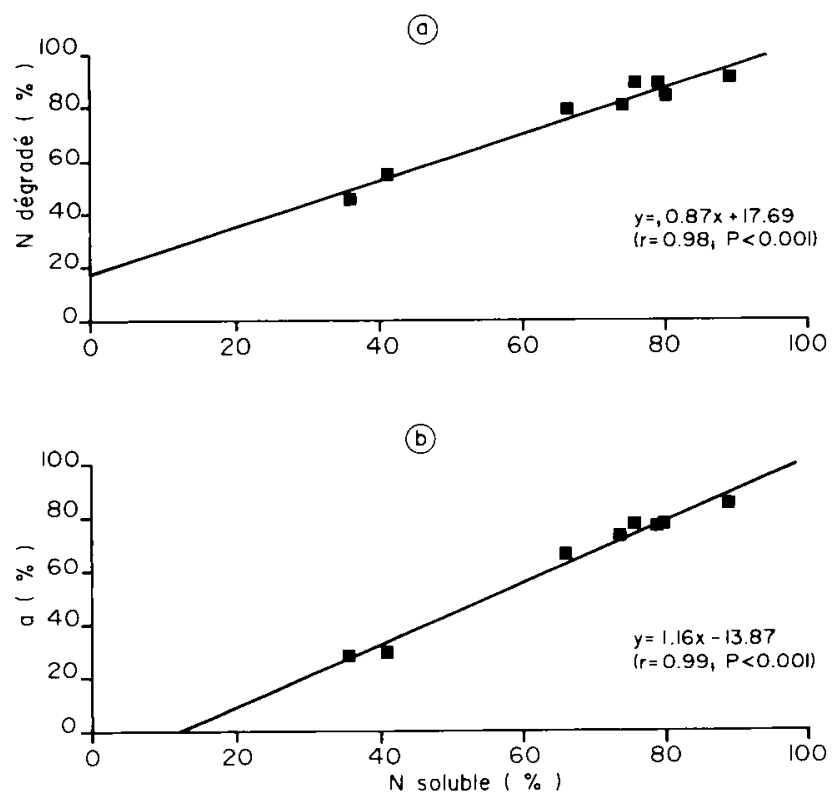

Fig 2. Relations entre la solubilité de l'azote in vitro et (a) : la dégradation in sacco de l'azote, après $2 \mathrm{~h}$ d'incubation dans le rumen et (b) : le coefficient a des cinétiques de dégradation de l'azote (cf tableau III).

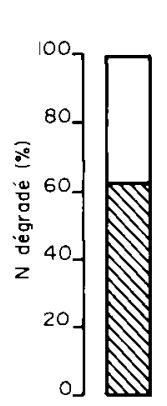

Grilles (mm) 5 (a)

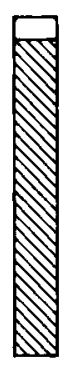

1.5

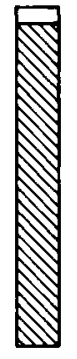

1 (b)

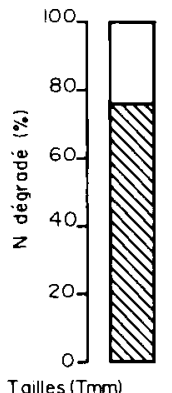

Tailles (Tmm)

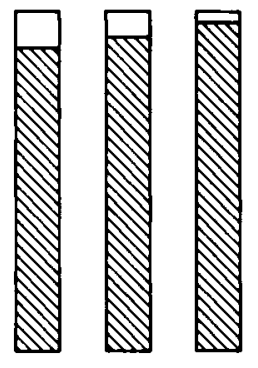

$0.25<T<0.75$

$$
T>1 \quad 0.75<T<1 \quad 0.10<T<0.25
$$

Fig 3. Dégradation de l'azote (N) du lupin dans le rumen ( $B$ ) et l'intestin ( $\square$ ) en fonction de la grille de broyage (a) et de la taille des particules (b). 


\section{Estimation de la valeur PDI du lupin}

Les estimations des teneurs $P D I$ sont compilées dans le tableau IV. Pour un broyage grossier, les valeurs ( $\mathrm{g} / \mathrm{kg} M S$ ) PDIN et PDIE obtenues sont de 291 et 219 respectivement. Sur un lupin broyé aux grilles de 1,5 et $1 \mathrm{~mm}$, les teneurs $P D I$ sont diminuées de 18,9 à $20,3 \%$ pour les PDIN et de 55,3 à $58,4 \%$ pour les PDIE. Les particules de lupin ayant une taille supérieure à $1 \mathrm{~mm}$ ont des valeurs PDIN et $P D I E$ de 267 et $166 \mathrm{~g} / \mathrm{kg}$ MS; lorsque la taille des particules est réduite, ces valeurs baissent de 8,9 et $30,7 \%(0,75<T<$ 1), 11,2 et $38,6 \%(0,25<T<0,75)$, et 13,9 et $48,8 \%(0,10<T<0,25)$ respectivement.

\section{DISCUSSION}

Pour la plupart des auteurs, la solubilité des protéines est la principale caractéristique déterminant leur dégradation dans le rumen (Crawford et al, 1978; Stern et Satter, 1984). Cette solubilité dépend princi-

Tableau IV. Estimation des valeurs PDI $(\mathrm{g} / \mathrm{kg}$ MS) du lupin en fonction de la grille de broyage et de la taille des particules.

\begin{tabular}{|c|c|c|c|}
\hline Paramètres & $P D I A$ & $P D I N$ & PDIE \\
\hline \multicolumn{4}{|c|}{ Grilles de broyage $(\mathrm{mm})$} \\
\hline 5,0 & 161 & 291 & 219 \\
\hline 1,5 & 29 & 236 & 98 \\
\hline 1,0 & 21 & 232 & 91 \\
\hline \multicolumn{4}{|c|}{ Tailles des particules (Tmm) } \\
\hline $1,00<T$ & 103 & 267 & 166 \\
\hline $0,75<T<1,00$ & 47 & 243 & 115 \\
\hline $0,25<\mathrm{T}<0,75$ & 33 & 237 & 102 \\
\hline $0,10<\mathrm{T}<0,25$ & 15 & 230 & 85 \\
\hline
\end{tabular}

palement du $\mathrm{pH}$, de la force ionique, du type de solvant et de la température (Wohlt et al, 1973; Blethen et al, 1990). D'après Duranti et Cerletti (1979), les protéines de la graine de lupin sont essentiellement composées de globulines : $87,2 \%$ (solubles dans les solutions salines) et d'albumines : $12,8 \%$ (solubles dans l'eau), ce qui explique le choix de la solubilité en milieu minéral comme index rapide de la dégradabilité des MA de cette graine. Pour le lupin, la solubilisation de l'azote est d'autant plus élevée que le broyage est fin. Les valeurs que nous enregistrons pour les échantillons broyés aux grilles de 1,5 et 1 $\mathrm{mm}$, eg, 75,5 et $78,8 \%$, s'écartent peu de celle qui est donnée par Vérité et Sauvant (1981) pour une graine broyée à la grille de $0,8 \mathrm{~mm}$, soit $81 \%$. Une liaison est trouvée entre la solubilité, la dégradation in sacco de l'azote $(2 \mathrm{~h})$ et le coefficient $a$ des cinétiques de dégradation. Une régression linéaire entre la solubilité en milieu tampon et la disparition in situ de l'azote a été également signalée par d'autres équipes pour différents aliments, eg, graines et tourteaux de soja, avoine, blé (Stern et Satter, 1984; Madsen et Hvelplund, 1985). Les valeurs de $D g N$ obtenues après broyage du lupin aux grilles 5 , 1,5 et $1 \mathrm{~mm}(62,5,93,0$ et $94,9 \%$ respectivement) sont très proches de celles trouvées pour cette graine dans la bibliographie. Freer et Dove (1984) notent que la $D g N$ in sacco est de $71,0 \%$ pour le lupin broyé grossièrement et de $90,0 \%$ pour un échantillon broyé finement. Par ailleurs, Sauvant et al (1987) et Andrieu et al (1988) estiment la $\mathrm{DgN}$ du lupin passé à la grille de $0,8 \mathrm{~mm}$ à $95,0 \%$. Toutefois, des données légèrement différentes sont mentionnées par d'autres auteurs; c'est ainsi que Valentine et Bartsch (1988) obtiennent une $D g N$ de $81,9 \%$ environ pour un lupin broyé à la grille de $1 \mathrm{~mm}$. De notre expérimentation, il ressort que le broyage fin a pour effet: 
- d'augmenter la fraction soluble (a) et rapidement dégradable des MA, ce qui est en accord avec les essais de solubilisation in vitro;

- d'accroître la vitesse de dégradation (c) de la fraction (b) lentement dégradable. II convient de noter que l'incidence du broyage sur la dégradabilité des MA varie avec la nature de l'aliment. En effet, d'expérimentations réalisées in situ par d'autres équipes (Nordin et Campling, 1976; Thomas et al, 1988), il ressort que la dégradation des protéines alimentaires du blé, de l'avoine, du maïs et de l'orge par la microflore ruminale est largement influencée par la qualité du broyage. Ces auteurs notent que l'attaque bactérienne est plus rapide et plus intense lorsque le broyage est fin (grille de $2 \mathrm{~mm}$ ), que lorsqu'il est grossier (grille de $6 \mathrm{~mm}$ ). Toutefois, Ehle et al (1982) n'ont pas pu mettre en évidence de relation entre la cinétique de dégradation des MA du tourteau de soja dans la panse et la taille des particules.

Les quantités de MA réellement indigestibles dans l'ensemble du tractus digestif ne sont significativement modifiées ni par la nature du broyage, ni par la taille des particules du lupin. Les teneurs en MA résiduelles dans les sachets mobiles après séjour dans le rumen et transit dans l'intestin sont très faibles, en moyenne $0,2 \%$. Des valeurs tout à fait comparables sont données pour la graine et le tourteau de soja (Peyraud et al, 1988). Un broyage grossier protège donc les MA de la digestion bactérienne dans les réservoirs de fermentation et, par voie de conséquence, augmente la part d'azote alimentaire qui parvient à l'intestin et qui disparaît ensuite au cours du transit. Dans cet essai, la digestibilité intestinale apparente des MA non dégradées dans le rumen est très importante : 96,1-99,5\%, alors que pour cette graine, la valeur de digestibilité réelle dans l'intestin grêle n'est que de $60 \%$ (Vérité et al, 1987). Pour le broyage à la grille de $1 \mathrm{~mm}$, les valeurs PDIN et PDIE $(\mathrm{g} / \mathrm{kg}$ MS) que nous obtenons sont proches de celles rapportées par Andrieu et al (1988) dans les tables de I'INRA pour un lupin broyé à la grille de $0,8 \mathrm{~mm}$, à savoir 232 vs 230 et 91 vs 82 respectivement; toutefois lorsque le broyage est réalisé aux grilles de 1,5 et $5 \mathrm{~mm}$, les teneurs PDI sont augmentées de 1,7 et $25,4 \%$ environ pour les PDIN et de 7,8 et $140,6 \%$ pour les PDIE.

De cette expérimentation réalisée in situ, il ressort qu'un broyage grossier, comparativement à un broyage fin, protège les MA du lupin de l'attaque bactérienne ruminale, sans toutefois affecter leur digestion dans l'ensemble du tube digestif, ce qui améliore théoriquement la valeur azotée de la graine.

\section{REFERENCES}

Andrieu J, Demarquilly C, Sauvant D (1988) Tables de la valeur nutritive des aliments. In: Alimentation des bovins, ovins et caprins (Jarrige R, ed), INRA Publ Paris, 351-434

Anonyme (1971) Méthodes d'analyses des aliments pour les animaux. JO Communautés Eur 20 déc 1971

Belyea RL, Martz FA, Mbagaya GA (1989) Effect of particle size on alfalfa hay on intake, digestibility, milk yield and cell wall of dairy cattle. J Dairy Sci 72, 958-963

Blethen DB, Wohlt JE, Jasaitis DK, Evans J (1990) Feed protein fractions: relationship to nitrogen solubility and degradability. J Dairy Sci 73, 1544-1551

Crawtord RJ, Hoover WH, Sniffen CJ, Crooker BA (1978) Degradation of feedstuff nitrogen in the three rumen vs nitrogen solubility in three solvents. J Anim Sci 46, 1768-1775

Dagnélie $P$ (1975) Théorie et méthodes statistiques. Vol 2, Presses Agron, Gembloux, $2^{\theta}$ edn, 245-250 
Duranti M, Cerletti P (1979) Amino acid composition of seed proteins of Lupinus albus. $J$ Agric Food Chem 27, 977-978

Ehle FR, Murphy MR, Clark JH (1982) In situ particle size reduction and effect of particle size on degradation of crude protein and dry matter in rumen of dairy steers. $J$ Dairy $S c i$ $65,963-971$

Eliman ME, Orskov ER (1984) Factors affecting the outflow of protein supplements from the rumen. 2. The composition and particle size of the basal diet. Anim Prod 39, 201-206

Eliman ME, Ørskov ER (1985) Factors affecting the outflow of protein supplements from the rumen. 3. Effects of frequency of feeding, intake of water induced by the addition of sodium chloride, and particle size of protein supplements. Anim Prod 40, 309-313

Emile JC, Huguet L, Hoden A, Malterre C, Micol D (1988) Sweet lupin seeds for dairy cows and young bulls feeding. In: Proc Fifth Int Lupin Conf (Twardowski T, ed), Poznan, Poland, 383-395

Freer M, Dove H (1984) Rumen degradation of protein in sunflower meal, rapeseed meal and lupin seed placed in nylon bag. Anim Feed Sci Technol 11, 87-107

Galyean ML, Wagner DG, Owens FN (1979) Corn particle size and site and extent of digestion by steers. J Anim Sci 49, 204-210

Galyean ML, Wagner DG, Owens FN (1981) Dry matter and starch disappearance of corn and sorghum as influenced by particle size and processing. J Dairy Sci 64, 1804-1812

Grenet E (1970) Taille et structure des particules végétales au niveau du feuillet et des fèces chez les bovins. Ann Biol Anim Biochim Biophys 10, 643-657

Guillaume B, Otterby DE, Linn JG, Stern MD, Johnson DG (1987) Comparison of sweet white lupin seeds with soybean meal as a protein supplement for lactating dairy cows. $\checkmark$ Dairy Sci 70, 2339-2348

Hove E, King $S$ (1978) Composition, protein quality, and toxins of seeds of the grain legumes Glycine max, Lupinus spp, Phaseolus spp, Pisum sativum and Vicia faba. NZ $J$ Agric Res 21, 457-462

Madsen J, Hvelplund T (1985) Protein degradation in the rumen $A$ comparison between in vivo nylon bag, in vitro and buffer measurements. Acta Agric Scand Suppl 25, 103-124

Michalet-Doreau $B$, Vérité $R$, Chapoutot $\mathbf{P}$ (1987) Méthodologie de mesure de la dégradabilité in sacco de l'azote des aliments dans le rumen. Bull Tech CRVZ Theix 69, 5-7

Nordin M, Campling RC (1976) Digestibility studies with cows given whole and rolled cereal grains. Anim Prod 23, 305-315

Ørskov ER, McDonald I (1979) The estimation of protein degradability in the rumen from incubation measurements weighted according to rate of passage. J Agric Sci (Camb) 92, 499-503

Peyraud JL, Genest-Rulquin C, Vérité R (1988) Mesure de la digestion de l'azote des aliments dans l'intestin des ruminants par la méthode des sachets mobiles. Reprod Nutr Dev 28, 129-132

Sauvant D, Aufrère J, Michalet-Doreau B, Giger $S$, Chapoutot P (1987) Valeur nutritive des aliments concentrés simples : tables et prévisions. Bull Tech CRVZ Theix 70, 75-89

Stern MD, Satter LD (1984) Evaluation of nitrogen solubility and the dacron bag technique as methods for estimating protein degradation in the rumen. $J$ Anim Sci 58, 714-724

Thomas EE, Turnbull GW, Russell RW (1988) Effect of particle size and steam treatment of feedstuffs on rate and extent of digestion (in vitro and in situ). J Anim Sci 66, 243-249

Thomson DJ, Beever DE (1980) The effect of conservation and processing on the digestion of forages by ruminants. In: digestive physiology and metabolism in ruminants (Ruckebush $Y$, Thivend $P$, eds) MPT Press limited Falcon House, Lancaster, England, 291-308

Valentine SC, Bartsch BD (1988) Degradation of dry matter, crude protein, fat, crude fibre and nitrogen free-extract in milled barley and lupin grains incubated in nylon bags in the rumen of dairy cows. J Agric Sci (Camb) 110, 395-398

Vérité R, Demarquilly C (1978) Qualité des matières azotées des aliments pour ruminants. In : La vache laitière INRA Publ, Paris, 143158

Vérité R, Michalet-Doreau B, Chapoutot P, Peyraud $J L$, Poncet $C$ (1987) Révision du sys- 
tème des protéines digestibles dans l'intestin (PDI). Bull Tech CRZV Theix 70, 19-34

Vérité R, Sauvant D (1981) Prévision de la valeur nutritive azotée des concentrés pour les ruminants. In: Prévision de la valeur nutritive des aliments des ruminants. INRA Publi, Paris, 279-296

Wohlt JE, Sniffen CJ, Hoover WH (1973) Measurement of protein solubility in common feedstuffs. J Dairy Sci 56, 1052-1057 